International Journal of Child, Youth and Family Studies (2014) 5(1): 24-46

\title{
LISTENING FOR THE VOICES OF RESILIENCE: A GROUP OF ADOLESCENTS' EXPERIENCES WITH A SUICIDE PREVENTION EDUCATION PROGRAM
}

\section{Chelsea K. F. Ohlmann, Janelle L. Kwee, and Robert Lees}

\begin{abstract}
Adolescents' experiences in a grassroots, school-based suicide prevention education group in British Columbia, Canada, were investigated in response to anecdotal observations of the group's strong positive impact. In the Alive group, at-risk high school students, 15 to 18 years of age, learned to support each other, became literate in mental health issues, and created and performed presentations on suicide prevention to peers. The authors employed the qualitative method of the Listening Guide to explore the participants' experiences of this program. Participant narratives reveal voices of vulnerability (including voices of not knowing, disconnection, and silence - primarily in reference to past experiences of suicidality and depression), and of resiliency (including voices of knowing, connection, altruism, and protection). Through their experience of the Alive group, the participants developed and strengthened inner and interpersonal resources of resilience and knowledge of themselves and others. Motivated by their own healing, they articulated a desire to help and impact others with what they had learned.
\end{abstract}

Keywords: suicidality, resilience, Listening Guide, suicide prevention education

Acknowledgements: This research was supported by the Social Sciences and Humanities Research Council. The authors would also like to acknowledge the assistance of Jennifer Decker, the Research Assistant for the present research, who had a significant role in the analysis process and implementation of the Listening Guide.

Chelsea Ohlmann, M.A. (the corresponding author) is a graduate of the Master of Arts Counselling Psychology program at Trinity Western University, 7600 Glover Road, Langley, B.C., Canada, V2Y $1 Y 1$ and has worked as a youth suicide prevention therapist in Mission, B.C. Phone: (778) 292-0142. E-mail: Chelsea.ohlmann@gmail.com

Janelle Kwee, Psy.D. is an Assistant Professor in the Master of Arts Counselling Psychology program at Trinity Western University, 7600 Glover Road, Langley, B.C., Canada, V2Y 1Y1. E-mail: janelle.kwee@twu.ca

Robert Lees, Ph.D. is a Community Psychologist for the Ministry of Children and Family Development of British Columbia, 8978 School Street, Chilliwack, B.C., Canada, V2P 4L4. E-mail: robert.lees@gov.bc.ca 
Recently, within both the medical and psychological fields, attention on effectiveness research in prevention has increased (Marchand, Stice, Rhode, \& Becker, 2011). Within psychology, there has been a growing emphasis on health promotion and well-being, which directs the emphasis away from a problem-focused orientation. This shift represents a move from tertiary prevention, or targeting treatment towards those who are already experiencing symptoms of a disease, to primary and secondary prevention, defined respectively as preventing disorder onset in the general population and preventing further onset of a problem among populations at elevated risk (Marchand et al., 2011).

Research and practice in the area of suicidality has also experienced a shift towards prevention. This shift has built upon the tenet that the traits or abilities an individual possesses have a protective influence with respect to negative risk factors. These internal protective factors and the process of their development are often referred to as resiliency. Johnson, Wood, Gooding, Taylor, and Tarrier (2011) conceptualize resiliency to suicide as "a perception or set of beliefs which buffer individuals from suicidality in the face of stressors or risk factors" (p. 563). Everall, Altrows, and Paulson (2006) define resiliency as "an adaptive process whereby the individual willingly makes use of internal and external resources to overcome adversity or threats to development" (p. 462). There appears to be a consensus in research literature that resilience is not a fixed attribute or outcome, but rather is a multi-dimensional and continually changing process (Everall et al., 2006; Richardson, Neiger, Jensen, \& Kumpfer, 1990; Rutter, 1987, 2001; Ungar, 2005). Resiliency has been connected to the area of suicide prevention in numerous recent studies (Everall et al., 2006; Johnson et al., 2011; Nrugham, Holen, \& Sund, 2010). In a narrow sense, resilience has come to mean the individual capacities, behaviours, and protective processes associated with health outcomes despite exposure to a significant number of risks. However, Ungar (2005) cautions against a purely individual approach to resiliency, stressing that it is complex, and that youth, their caregivers, and their communities travel on multiple pathways toward health. Therefore, it is essential to consider the experience of at-risk youth in developing and enacting resiliency when we examine the suicidology literature. For the present study, resiliency is not solely conceptualized as the development and utilization of internal coping resources by an individual, but also as the complex process emphasized by Ungar, one that individuals, their families, and their communities engage with to travel towards and achieve healthy outcomes.

The shift in focus towards prevention and the increasing emphasis on resilience is a much-needed approach when one considers the important concern of suicide within youth populations. According to a nationwide survey of students between Grades 9 and 12 in the United States (Hooven, Herting, \& Snedker, 2010), 15\% of respondents had seriously considered attempting suicide, $11 \%$ had made a specific plan, and $7 \%$ had attempted suicide within the 12 months preceding the study. In Canada, suicide is the second leading cause of death among youth between 12 and 18 years of age (Rhodes, Bethell, \& Bondy, 2006; Skinner \& McFaull, 2012). The statistics are comparable in the 
Province of British Columbia. According to a study by the McCreary Centre Society involving over 50,000 teenagers, suicide is the second leading cause of adolescent death in British Columbia, and in 2008, 12\% of students surveyed had seriously considered suicide and 5\% had attempted suicide (Smith, Stewart, Peled, Poon, \& Saewyc, 2009). Although self-reported suicide ideation and attempts among youth may have declined recently (16\% in 2003 to 12\% in 2008; 7\% in 2003 to 5\% in 2008, respectively, according to Smith et al.), it is important to acknowledge recent evidence that suggests over the past 30 years, rates of suicide among Canadian adolescent girls have increased (Skinner \& McFaull, 2012).

School-based suicide prevention programs surfaced in the 1980s as a response to the significant increase in suicide rates that many Western industrialized nations experienced (Guo \& Harstall, 2002; White, Morris, \& Hinbest, 2012). Since the emergence of school-based suicide prevention, there has been a similar emphasis within the literature to evaluate the effectiveness of these programs in reducing suicidal ideation and completed suicides in the adolescent population. In turn, youth suicide prevention education programs have followed the emphasis on evaluating effectiveness and on prioritizing evidence-based practice highlighted in the literature, and many prevention programs are now predicated on what is deemed to be effective, that is, on what works. While research on effectiveness is valuable and necessary, an overemphasis on efficacy may increase the tendency of researchers to focus on evaluations from a quantitative perspective. Both qualitative and quantitative are valuable approaches of inquiry and offer unique perspectives when considering both the effectiveness of and experience of suicide prevention programs. Furthermore, research in the area of suicide prevention education has not reached a conclusive answer as to which prevention education efforts are most helpful. Guo and Harstall's (2002) systematic review of early suicide prevention programs concluded that "there is insufficient evidence to either support or not to support curriculum-based suicide prevention programs in schools” (p. iv).

Though some of the early school-based prevention education programs were examined with mixed results, the emerging focus on program evaluation led to more rigorous research and evaluation studies. For example, a more recent systematic review demonstrates similar results: Miller, Eckert, and Mazza (2009) found that of the 13 studies reviewed, five showed promising evidence for outcomes of statistical significance, and only two demonstrated strong evidence. Corcoran, Dattalo, Crowley, Brown, and Grindle (2011) conducted a systematic review of 17 studies and also reported mixed results. These authors state that in the studies that measured suicidal ideation at post-test, intervention group participants were slightly less likely to report suicidal ideation than control group participants, both at post-test and at follow-up. However, for studies that measured both suicidal and self-harm events and assessed the outcome later than immediately after the intervention, the intervention group was slightly more likely to have suicidal and self-harm events than the control group. The authors interpret these contradictory findings to mean that interventions are only slightly effective in reducing suicidality in adolescents, and that they may, in fact, increase suicidal and self-harm events over time. Despite this finding of Corcoran et al., other studies have suggested that some programs can lead to increased student knowledge and change unwanted attitudes, 
International Journal of Child, Youth and Family Studies (2014) 1: 24-46

without undesirable effects (Ciffone, 2007).

One response to the apparent lack of evidence is to call for more rigorous methodology in the effectiveness research and to increase the number of evidence-based suicide prevention programs in schools (Tompkins, Witt, \& Abraibesh, 2010). However, White et al. (2012) argue that the traditional evidenced-based practice and research focus "tends to obscure the complexity and dynamic nature of social learning, privileges a unitary and individualistic understanding of suicidal behaviour, and fails to account for the multiplicity of ways in which understanding suicide and ideas about prevention might be understood" (p. 346). For these reasons, an equally valuable response is to recognize that better understanding the components and mechanisms at work within suicide prevention programs is necessary to provide insight for decision-makers in determining future suicide prevention strategies (Guo \& Harstall, 2002). As White et al. (2012) suggest, the narrow range of methodologies that have been utilized to study school-based suicide prevention education initiatives, the lack of definitive conclusions, and the resulting questions that remain around suicide prevention set the stage for "alternative conceptualizations and fresh approaches to inquiry" (p. 344) of suicide prevention education programs.

Though there have been many attempts within the literature on suicidality to demonstrate effectiveness, there is little research on the adolescents' experiences of the intervention programs. Michael Ungar (2002) cautions against taking a top-down approach in understanding youth, as this tempts those in power positions to assume they understand the youth and as a consequence make decisions too quickly. Rather, he argues that a voice is missing from the literature pertaining to the field of interventions, specifically the voices of the youth themselves (Ungar, 2004). As we listen to the narrative of effectiveness within the suicidology literature, the lived experiences of the prevention program participants is starkly absent. Therefore, in the following paper, we aim to explore the insider accounts of the participants of one such suicide prevention education program. In a high school in British Columbia, an intervention program with high-risk adolescents experiencing suicidality appeared to result in notable improvements for its participants. Through learning about suicidality and presenting suicide education information to their peers, the participants seemed to have overcome much of their previously suicidal thoughts and behaviours and developed into purposeful and capable peer leaders. The perception of teachers and school counsellors that remarkable change occurred within the participants provided the basis for further investigation of the participants' experiences. Through exploring the thick narratives of their experiences, we intend to serve as a vehicle that allows their voices to be recorded in the suicide prevention literature.

\section{The Present Investigation}

\section{Background of the Alive group}

The Alive group was a youth-led suicide prevention education group sponsored by the F.O.R.C.E. Society for Kids’ Mental Health, a non-profit agency. The group was 
established to enhance training and skill development among at-risk youth. Through participation in the Alive group, the participants in this study provided support to each other and became literate in mental health issues, recognizing the signs and symptoms of emotional distress and developing the skills needed to reach out to peers. After learning about various mental health disorders and the connection between mental illness and suicide, the group members decided to focus on suicide prevention. The participants learned a model of responding to suicidal ideation called “Ask, Assess, Act”. As the participants experienced the value of learning this model and applying it to their own lives, they decided to create a presentation based on the model to share with other students and other schools. As a result, the group had a dual focus: first, to educate the members of the group and second, to reach other youth and teach them how to recognize signs of suicide risk in themselves and in their peers (Leuthardt, 2011). Because of the organic, student-led nature of the group's development, its goals and focus were strongly impacted by the personalities and passions of the members. Because of this grassroots development and the way that the structure of the group was adapted to fit the needs of the members, the goals and strategies of the Alive group evolved as the group journeyed together. For these reasons, looking at the participants' experiences may prove to be the most helpful approach to understanding the nature of the Alive group.

\section{Participants}

The participants in this study included five high school students between 15 and 18 years of age. Some of the students had been identified by school staff as being at risk for self-harm or suicide; other students had a personal connection with suicide. Four of the five participants in the present study initially joined what they referred to as a "girls' group” in Grade 10, which was intended to provide a supportive environment for these students. The students decided that they wanted to make a difference for others and transitioned into a suicide prevention education group, which they named Alive. The participants who had been part of the group from the outset had been in the group for three years; one participant had been in the group for one year.

The teachers and mental health workers involved in the group's leadership observed remarkable changes in the participants over the course of their involvement in the group, and because of this the group was identified for the present research. The participants involved in this study were high school students who were part of a preexisting, school-based intervention group, which had become a suicide prevention education group, called Alive. The complex lived experiences of the participants from this group may inform the research and practice of other similar suicide prevention programs.

\section{Method}

The Listening Guide (Gilligan, Spencer, Weinberg, \& Bertsch, 2003) was chosen in order to more fully understand the participants' experiences of this suicide prevention education group. A relational method, such as the Listening Guide, is intended to recognize the layered nature of psychological processes and to interpret psychological 
behaviour within a relational and cultural context. Carol Gilligan (1982) laid the foundation for the present method through her work on identity and moral development. The Listening Guide is based on psychoanalytic theories, with an emphasis on the layered nature of the psyche, as well as on relational and feminist psychologies, wherein one's sense of self is inseparable from one's relationships and culture (Gilligan et al., 2003). The Listening Guide involves reviewing the multiple voices within the participants’ transcripts and exploring how the voices interact within their expressed experience to convey, or to hide, their inner world. Mikel Brown (1997) describes how the Listening Guide provides a way “to trace the movement in girls' understanding of themselves and others as they take in the voices around them, both appropriating and resisting the different perspectives on relationships” (p. 686).

This qualitative method is best used when the research question requires listening more deeply both to a person's expressed experience and to the contexts in which their experiences occur (Gilligan et al., 2003). For instance, Gilligan and Machoian (2002) demonstrate that the Listening Guide offers a fresh approach to the exploration of adolescent girls' suicidality. These authors argue that in early adolescence, girls' resilience is at risk, and their peak susceptibility to suicidality reflects a fight for relationship. Interpreted in this light, suicidality can be viewed as a way of "enacting the hope that someone does care about them and will listen and take them seriously" (Gilligan \& Machoian, 2002, p. 323). Gilligan and Machoian further suggest that girls may learn to speak the language of violence in order to be taken seriously. Through a relational interpretation of her suicidality, the authors understood one participant's suicidality as "an active resistance to disconnection; as an active fight for voice” (p. 333). In this way, an adolescent girl's suicidality can be seen, they state, as a "complicated hope for relationship” (p. 335), demonstrating that a relational interpretation of suicidality can offer new understandings and unique perspectives.

With a strong relational component, the Listening Guide intentionally brings researchers into relationship with participants through making one's experiences and interpretive lens explicit. The process of making the researcher's lens explicit will be further elaborated when we describe the implementation of the Listening Guide. The structure of the method is flexible rather than a set of prescriptive rules. For these reasons, the Listening Guide empowers participants, enabling them to impact how researchers listen, how researchers ask questions, and how the participants' expressed experience is interpreted. The researcher listens first to the participants' voices before looking for answers to the research question (Gilligan et al., 2003). The specific process of implementing the Listening Guide will be described in more detail subsequently in the present section.

The Listening Guide was expected to cast new light on the experiences of the Alive group members, interpreting their experiences within the context of adolescent female development and within the cultural context in which they live. This method involves listening deeply to the participants' experiences, capturing not just what they say directly, but also the meaning and context behind their words. Exploring meaning and context is a reflexive process that thickens as the relationship develops between the 
researcher, the data, and the participants. For example, in the present study, one participant describes the group as being "not a school activity". In order to more fully understand what is meant by these words, it is essential to explore this participant's experience of school and of the group. In this instance, the participant experiences school as a disconnecting, isolating, and impersonal place. Conversely, she experiences the suicide prevention group as a place where her individuality is valued and she can use her voice to help shape the direction of the group. Considering the context behind her words, therefore, more fully explicates what she means when she describes the group as "not a school activity”. Due to the dynamic interaction between the experiences of suicidality, the unique challenges of being an adolescent, and the complex and multi-dimensional ways that individuals experience suicide prevention and education, the Listening Guide was identified as an appropriate way to capture the participants' experiences in order to address the research question of the present study.

\section{Data Collection}

The Research Ethics Board at Trinity Western University, as well as the relevant school district, approved the research study. The consent process involved demonstrating the rationale for the study, requiring all participants to receive parental consent, and offering opportunities to debrief their experiences with the study. Some might suggest that it could be harmful for the participants to be asked about their experiences with suicidality. However, these potential participants have been taking part in the suicide prevention group for over a year and each individual had been delegated their own piece in the presentation, which involved sharing how they have been personally touched by suicide. In the focus group, students discussed the experience of sharing: "I really liked that we got to tell our stories, this was a real healing process for me", and "it was a really nice feeling to be heard, it made us feel like we were looked up to". Furthermore, research has demonstrated that talking about suicide does not increase an individual's suicide risk (Ciffone, 2007).

The researchers gathered data through semi-structured interviews, following the Listening Guide protocol. Students and parents completed a written informed consent questionnaire, and interviews of 45 to 90 minutes duration took place either at the individual participant's school or at an outside office. At the beginning of each interview, participants were invited to engage the interviewer with questions about the process, and informed consent and confidentiality were reviewed. The researchers implemented the interview protocol with flexibility, which involved responding to markers of intensity or conflict, asking further about questions with strong emotional content, clarifying meaning, and building rapport throughout the interview. Maintaining openness serves to explore the participants' experiences in greater detail, eliciting more richness and depth in the interview data. The interviews were video recorded and systematically transcribed concurrent with the data collection process. The transcribed interviews were anonymized and all identifying information and names were changed. The researchers had access to the videos and transcripts throughout the analysis process. 
At the end of each interview, participants had the opportunity to debrief. After all the interview questions had been asked, the same interviewer asked if there was anything else the interviewee would like to talk about and offered to connect them with any other necessary supports (e.g., counselling). Though the interviewer was available for as much time as the participants needed to debrief, participants for this study utilized only a few minutes and none of the participants seemed to have been impacted in a negative way by the interview. In fact, many participants spoke of the value of reflecting on their experiences. A focus group was also carried out with the participants after the interviews were complete. The goal was to provide a forum for the youth to reflect on the experience of participating in the study and share with each other what they had learned. The primary researcher was also available to the participants by phone for the duration of the study and up to four weeks after the study's completion.

\section{Implementing the Listening Guide}

The Listening Guide was implemented according to the protocol and guidelines outlined by Gilligan et al. (2003). Sequential sessions of listening help the researcher glean distinct aspects of one's experience, co-occurring voices, and the relationship between voices (Gilligan et al., 2003), which adds depth and richness to the interpretation of the participant's expressed experience. A brief outline of the sequential listenings is included in the following section, and this section serves as the description of the data analysis protocol for the present study; for a more detailed description of the Listening Guide method, see Gilligan et al. (2003). It is important that the steps be implemented with flexibility, paying attention to the iterative and non-linear nature of qualitative analysis. The analysis process was completed by a research team, which included the primary researcher (Chelsea Ohlmann), Dr. Janelle Kwee (the research supervisor), and Jennifer Decker (the research assistant). Any further references to the research team refer to these individuals.

Step one: Plot and listener's response. Step one involves listening for the plot, including the landscape, multiple contexts of the story, what is happening (what, when, where, with whom, and why), repeated images or metaphors, dominant themes, contradictions and absences, the social context of the story, and the research environment. Listeners also pay attention to their own response to the narrative, including thoughts, feelings, what touches them, where they feel connected or disconnected, their respective social positions, and how these responses impact their understanding of the person. Identifying personal responses enables researchers to separate their experiences from those of the participants. The researchers completed this step individually and wrote down observations; these observations were then shared verbally as a team and the primary researcher compiled the varied perspectives. Acknowledging one's interpretive lens is embedded within the analysis process and in conversations with the research team; the interpretive lens does not come into the results explicitly because it is so embedded in the process. For instance, one participant referred to the importance of her relationship with her cat and she spoke of "having her cat to talk to". The varied perspectives of the research team caused some to see this as evidence of disconnection, while others saw this as indicative of a connective experience. As we made our lenses explicit, we had 
International Journal of Child, Youth and Family Studies (2014) 1: 24-46

meaningful conversations that led to new and deeper understandings of this participant's experience. Though the listener's response and interpretive lens is too complex to tease out separately, it is very much embedded within the results.

Step two: I poems. The second listening helps the researcher listen to the participant's first person voice, capturing what the participant may not have said directly but that is nonetheless central to the meaning. When the participants use the word $I$, they are talking most directly about themselves, so tuning into first person voices enables the listener to attend to what the participants know about themselves. To construct the "I poem", each first person $I$ was identified within the passage, along with the verb and any important accompanying words. Maintaining the original sequence of the text, we pulled out each underlined phrase and arranged each phrase on a separate line. The I poem picks up on an associative stream of consciousness that runs through each participant's narrative, and the stanzas reflect shifts in meaning or voice.

Step three: Listening for contrapuntal voices. Listening for multiple voices enables the researcher to discover several different layers within a person's expressed experience. The concept of contrapuntal voices is based in musical counterpoint, where two or more melodic lines, which have independent rhythm and contour, move in relationship with one another. Listening sequentially for various contrapuntal voices within participants' expressed experience allows the researcher to access and describe diversity within unity. Identifying harmony and discord between the voices illuminates the ontological complexity of the participants' experiences with coherence and detail.

Each contrapuntal voice was identified in a separate listening. The essential qualities of each voice were identified by critical markers, such as "references to identifying with others" as a marker for the voice of connection, for example. The use of markers rather than static definitions for each voice is consistent with the iterative and reflexive nature of the research process. In this process, the research team revises, expands, and continually nuances their understanding of each voice. Relationships between the contrapuntal voices and the first-person voice emerged, revealing whether the voices were in harmony with or in opposition to one another. As each voice should illuminate a meaningful aspect of the text, the researchers continued to identify voices until the meaning of the text was most fully represented by the identified voices. Descriptions and examples of each contrapuntal voice are elaborated in the results.

Due to the emphasis of the relational nature of the method, each interview was analyzed and voices were identified by at least two members of the research team. This collaborative process enabled the research team to discuss similarities and differences between participants, to consider how the person of the researcher impacts the process of analysis, and to elicit more richness and depth in the understanding of the participants' narratives. The research team read the first interview and identified preliminary voices that were most clearly heard in the first interview. For instance, it was very clear that at times the first participant spoke in a knowing, confident voice, while at other times she spoke with hesitancy or spoke directly about a lack of knowledge. As a result, we began by coding for the "knowing” and "not knowing" voices. Each voice was coded in a 
separate listening and we continued to identify additional voices until it appeared that the meaning behind a participant's words was captured. As we continued to identify voices, the voices seemed to form two categories: voices of resilience and voices of vulnerability. The voices of resilience include knowing, connection, altruism, and protection. The voices of vulnerability include not knowing, disconnection, and silence.

After the voices were identified, the researchers used the same process to code for voices in subsequent interviews, listening for similarities and differences between participants. When differences were noted, the researchers identified either a new voice, or the nuances of how a particular voice varied between interviews. For instance, the voice of silence was originally identified in the first interview as we heard the participant speak about not being able to say what was on her mind. In another interview, the participant also evidenced self-judgement as she spoke. As a team we determined that this self-judgement or self-silencing fit within the voice of silence, although it was qualitatively different from what we had heard previously. In this way, the researchers implemented the Listening Guide reflexively, taking into account the individual experience of each participant and the reactions of the researchers.

Step four: Composing an analysis. Once all interviews had been listened to multiple times and the voices had been identified, the primary researcher composed an analysis for each participant, synthesizing the identified voices with what had been learned about the participant. For example, one participant shares in a knowing voice that her story is not something to be ashamed of, yet she follows her statement of knowing with a voice of not knowing - saying "I guess" - thereby making her knowing voice sound more hesitant and doubtful. Bringing the voices of each interview back into relationship with one another aids in retaining the complexity of the participants' experiences and enables us to access the diversity in their words that may have been missed had we merely analyzed by theme. In short, steps one through three are compiled at this point, creating an overall narrative of the participant's experience. Multiple interviews were then looked at in relation to one another, and the researchers looked for similar and contrasting voices across participants. This helps the researchers to explore the participants' individual and collective experiences of the suicide prevention education program and how it impacted their resilience. The following section includes an outline of the identified voices.

Focus group. A focus group was held with the participants after all the interviews were complete. The purpose of the focus group was to provide greater insight into the context of the participants' experience as a social unit and to give them another opportunity to debrief and reflect upon their experience of the study. Though the participants had the opportunity to reflect on their experience of the group within the individual interviews, the focus group gave them the opportunity to reflect together on what the group had been like, creating a shared meaning of their experience. Furthermore, the focus group provided a credibility check as the researchers shared the voices that had been identified and the participants were able to respond. Overall, the participants expressed agreement with what had been heard and summarized by the researchers. 
International Journal of Child, Youth and Family Studies (2014) 1: 24-46

\section{Results and Discussion}

Although the purpose of the present study was to explore how resiliency was fostered through adolescents' experience of a suicide prevention and education program, we approached the analysis of the interviews with openness to what would emerge from the participants' voices. This practice is consistent with feminist research, as a fundamental tenet of the Listening Guide approach is to allow the participants to shape the methodology and analysis as the research develops (Gilligan et al., 2003). Through immersing ourselves in the interviews, it became apparent that the way the participants spoke about themselves and their experiences unfolded into two overarching themes: voices of vulnerability and voices of resilience. We review these voices, providing illustrations of how these themes emerged from the narratives.

\section{Voices of Vulnerability}

Voice of not knowing. The not knowing voice occurred as participants spoke with hesitancy, were expressing a lack of understanding or awareness, or were questioning their own voice or ability to know. Markers used to identify this voice include a manner of speaking that indicates hesitancy or uncertainty, participants speaking directly about their lack of knowledge, or implicit references to lack of knowledge.

The not knowing voice presented within the participants' expressed experience through questioning their own voice or experience. As the participants were speaking, there were instances in which qualifying statements such as "kind of", "I guess", "I think", and "I don't know" preceded or followed what they were saying, making their knowing voice appear more hesitant. The not knowing voice also occurred as the participants were speaking about a lack of explicit or experiential knowledge. For instance, when talking about being depressed and suicidal, Jane shares, "When I was in that state, I didn't know how I felt and I didn't know really what the signs were." Exemplifying experiential not knowing, Harper shares that even though she knew what to do, getting emotionally overwhelmed kept her from using her knowledge effectively. She states that at first, “you just freak out, you just say whatever's on your mind, like you don't know what to do, you don't sit back and like try to think about everything you know." Though learning the knowledge was the beginning of knowing, utilizing this knowledge effectively was not automatic.

Through the analysis process, we noticed that many of the participants directly and indirectly referred in their interviews to a society-based voice of not knowing, for example including references to cultural norms that reflect ignorance about suicide as well lack of understanding between adults and teenagers.

Voice of disconnection. The voice of disconnection was marked by references to distance, conflict, or superficiality within relationships, talking about being alone, or an inability to connect to others. 
For many of the participants, references to the voice of disconnection were prominent in their before and after narrative of involvement in the Alive group, describing a feeling of vulnerability and isolation prior to experiencing a sense of belonging or connection through involvement in the group. Jane shares about her life before the group: "I had nobody to talk to at all... I felt, like, really alone, like nobody was there anymore for me." Some participants spoke in a voice of disconnection when they described specific relationships, such as with their parents or adults in the helping profession.

The voice of disconnection is also apparent as the participants talk about their experiences with mental illness. For example, Anne states, "The worst thing about depression is I did really questionable things and really terrible things to my family during those years, and it's really hard to reclaim the relationships." While the sense of isolation and disconnection not only influenced the development of mental health concerns, these same issues also contribute to further disconnection.

Voice of silence. Markers of the voice of silence include evidence of any time a participant felt unable to say what was on her mind or kept a secret, references to silencing in the world, or internalized self-judgment.

The voice of silence, commonly heard in references to relationships, often coincided with relational disconnection. Revealing a discrepancy between her internal feelings and expressed experience, Harper easily shares what she wants to say to a friend when he is suicidal, following her statement with "but I never say that to him.” A similar disconnect is evident as she speaks about her relationship with her mother: "I was so mad at her for doing that but I didn't wanna tell her."

Within the voice of silence, many of the participants referred to silence in an indifferent world, including stigmas against suicide and mental illness and a real or perceived inability for the participants to speak into the world. Anne's experience of the silence in the world includes an expectation that she would be judged due to her depression. Lucy elaborates further on the silence in the world as she shares about the reactions of some teachers upon hearing that she was in a suicide prevention group, and the "stigma that you don't talk about [suicide]." She continues to share about how suicide often gets "swept under the carpet." All of the participants demonstrated some aspect of stigma in the cultural status quo regarding the topic of suicide.

There was also evidence of internalized self-silencing, demonstrated by participants judging themselves based on stigmatization. For instance, Anne shares her own experience with depression and self-harm, and she follows up her words with "you know, this whole story" in a mocking, minimizing tone, further stating that she tends to devalue her story when she hears those of others.

Finally, we heard the voice of silence as participants related their experiences with helping professionals. Jane refers to the legal limits of confidentiality in counselling as silencing, inhibiting her comfort in sharing her feelings and thoughts about issues like 
International Journal of Child, Youth and Family Studies (2014) 1: 24-46

suicidality because sometimes counsellors “[can't] keep stuff a secret.” Anne further reflected on her felt experience of being silenced in a counselling setting: "Nothing's worse than trying to tell someone that you cut yourself or that you throw up to someone you don't even know that's, like, super old, and they're like [looking at their watch].” It appears that some elements of formal counselling made it more difficult for some participants to share.

Voice of literal silence. Though the concept of voice serves as a metaphor to understand one's dynamic relationship to oneself and the world, we also noted literal references to voice as expression and to silence as not speaking. A variation of the voice of silence that was heard in some interviews was a sense of being pressured to fake what one was feeling or thinking. This voice seemed to represent the actual living out of silence in their lives by purposefully hiding their authentic selves. Jane demonstrates this voice within her experience as she shares about feeling sad, yet faking a smile and pretending she was happy. Harper also demonstrates this literal silent voice as she shares about the discrepancy between what she was thinking and what she was actually saying to a friend.

\section{Voices of Resilience}

Voice of knowing. The markers denoting the voice of knowing include words that were emphatic, such as definitely; a tone of confidence, certainty, or purposefulness; or a sense that the participant had come to a new realization. The participants used the knowing voice when speaking about something that was important to them or something that was true for them. Listening to the participants' knowing voices, we had the clearest sense of hearing what was most representative of their internal experiences and the impact of their participation in the Alive group.

The knowing voice appeared in references to factual and inner knowledge gained within the Alive group. For example, Jane shares, "I've been more aware of people's actions and how they feel, and I can pick up when people feel depressed or suicidal." She further describes the confidence this knowledge has instilled in her: "I know what to do and how to help him." For Anne and Lucy, their knowing voices are used when they share how they have become more comfortable with themselves and owning their own experiences. Anne states that the group "definitely made it easier for me to be comfortable with my story." With a similar sense of confidence, Jane explains that being in the group has created a sense of certainty about who she is and who she wants to be in the future. She states, "I definitely would say me being in the group has made me a better person and has made me somebody who I want to be for the rest of my life."

Another aspect of the knowing voice that emerged included an intuitive sense of being able to access knowledge when it is needed and having a sense of the limits of knowledge. Lucy states that "being able to understand what you're supposed to do and then being able to put it into action are two totally different things because it is the most scary situation where your friend's like, I'm going to kill myself." Similarly, Harper shares that "in the moment it's hard to remember like you don't think of how to deal with it 
International Journal of Child, Youth and Family Studies (2014) 1: 24-46

calmly, you just freak out... so now I know how to deal with it without freaking out." These participants demonstrated a realistic and grounded sense of knowing, recognizing that suicidality is overwhelming, but at the same time, their knowledge can better equip them to handle these situations.

The knowing voice was also used when participants were speaking about being more settled in relationships and understanding others. For instance, Lucy notes that, "being in a group like this not only teaches you about suicide but teaches you that there's so many different levels to people."

Anne uses her knowing voice to share that she feels more independent and better able to handle her emotions. She states, "I was finally able to stand on my own and be happy" as a reference to how participation in the Alive group impacted her. In this way, the knowing voice connects with her ability to feel in control of her own emotions, rather than be controlled by them. From this place of knowing, she is able to share about what it is like to be depressed. She states, "The worst part of depression is not being able to control how you feel and not being able to be like, you know what, life sucks but I'm going to feel okay today. You just can't. You want to feel happy, but you just can't. It really sucks." Better understanding her own emotional experience, both now and in times of depression, helps her to be an empowered agent of her own change rather than a passive recipient of the emotions that previously dominated her experience.

The knowing voice also included evidence of the participants' abilities to set boundaries for themselves. For instance, Anne expresses a desire to continue helping others in her future. However, from her knowing voice, she expresses concern that if she does go into a helping profession, it might negatively impact her mood. In this way, the knowing voice is heard not as a naïve or idealistic desire to help others, but a desire to help with an awareness of how it would impact herself.

The voice of knowing also appeared in the form of giving advice about adolescents or about counselling. For instance, near the end of the interview Anne says, "I'm way younger than you, but I'm gonna give you some advice here. . . . the best counsellors are the counsellors that get to know you as a person first rather than get to know you as someone who's depressed." Harper also offers some advice about suicide prevention as she shares:

When it comes to suicide prevention, there's a lot of focusing on the people that commit suicide or have attempted, and the people that have lost but there's not much focusing on the people that are the people that they come to. So I was like, I know there's a lot of people that get in that situation and no one ever really focuses on that part of it.

Harper knows that because she felt unprepared to deal with her friends that experienced suicidal thoughts, there must be others out there who also need to know more about how to help their friends who experience suicidality. Based on her experience, she felt confident and sure about the recommendations she had for suicide prevention work. 
International Journal of Child, Youth and Family Studies (2014) 1: 24-46

Harper continues to offer advice as she speaks about the reason that the Alive group had such an impact. She shares, "I think the reason why we get through to them more is because we are their age...it hits closer to home for them." The importance of the peerto-peer education is further elaborated on within the voice of connection.

Lucy's voice of knowing sometimes takes on a unique quality; presented charismatically, her words have a sense of carrying a strongly felt message:

You don't have to tell everybody that you're feeling that way, but there's definitely other ways. You don't have to self-medicate yourself in order to feel better. You don't have to start cutting to feel better. You don't have to do any of those things. There's help. There's definitely help for you.

The purposeful sentences she has chosen to represent her knowledge demonstrate an ability not only to know, but an ability to persuasively communicate what she knows.

Voice of literal knowing. The literal knowing voice included participants' references to what they actually said and to being able to speak. When speaking in the literal knowing voice, it is clear that the participants intend to be listened to and are communicating in a way that demands to be heard.

Jane uses her literal knowing voice to relate being able to talk frankly to her parents. She also used this voice as she shared how in the group, she was able to talk and state her opinion. Anne told how the group enabled her to express what she was feeling. With a similar sense of literal knowing, Harper described the boundaries she has learned to establish in relationships. Harper demonstrates that though she is able to care for others in crisis, she is also able to create boundaries so that she does not lose herself in the process.

We heard the participants' voices of literal knowing as an ability to put flesh on their ability to know. Though many of their words exemplify metaphorical voices, these words are literal references to voice. In these moments, the participants demonstrated that they did not just gain knowledge; they also gained the ability to implement their knowing voice through powerful words and actions.

Voice of connection. The markers of the voice of connection include references to encounters with others, identification with others having similar traits or experiences, and any language that signifies having a close or connected relationship. Connection was a prominent aspect of all of the participants' experiences.

The voice of connection was strongly tied to the participants' experience of solidarity in the Alive group. Within the Alive group, the participants were able to share their stories and learn that they are not alone. Lucy describes her sense of connection in the group stating, "All other people don't connect with each other the way we connect, and it's really kind of amazing and empowering." In the Alive group, the participants experienced their connection with each other as novel and empowering. 
Sharing one's story; hearing the stories of others leading to increased understanding of oneself and others; representing an inextricable link between the experiences of connection; feeling a sense of knowing: all these are intrinsic to the connection voice. Participants referenced their experience of identification with others in the context of their presentations to other students about suicide and in the context of their relationships in the Alive group. Jane shares a story about a girl who, after listening to her story in the presentation, approached Jane and said, "Thank you so much, I understand now what's going on with me." Anne captures the mysterious felt sense of connection while sharing her story in a presentation: "Something I said really kind of made her feel something... I could feel that something within her connected to something that was said... it was just something about that whole moment, you know?" Referring to her experience in the group, Anne also mentions a realization she had after listening to the deeper issues others in the group experience: "Then you're like, oh, everyone goes through that." Being understood, or having someone identify with you, helped the participants to come to a place of knowing.

The absence of judgment is another aspect identified as part of the experience of connection. Lucy speaks about a particular presentation that was smaller and more intimate: "You could feel the connection between them, and it wasn't - like, nobody judged you." In this judgment-free environment, everyone had a voice and everyone was valued. For Jane, the sense of equality and teamwork she felt within the group was important. She states, "We usually work together on stuff, and we just try to make everything really equal, like when we voted for our name."

Trust was vital in this group because it enabled the participants to share without fear. Harper contrasts this experience of trust in the group with the norms of high school. Sharing within the context of the Alive group built trust with the members, which provided them the safety and support necessary to share their stories in the presentation. As Harper says, "Because I was with those people that were sharing the same amount of stuff it was like we would all share stuff with people we didn't even know. But it was because we were together that it was okay."

Through experiencing powerful connections within the group and at presentations, participants appeared to develop a desire for deeper connection in their lives. Lucy shares: "I've always kind of been more interested in people, what makes people tick, why do people think that way, and it's just kind of grown deeper." It also appeared that the participants developed greater empathy for others, with Anne revealing that, "I feel bad for people that are down, you know, because I've been there."

The participants also spoke about the power of identification experienced by having teenagers, instead of adults, share with other teenagers. Jane explains, "having a teenager talk about [suicide] to another teenager is way more impact to a person than having an adult." In a voice laden with both knowing and connection, Anne says, "I think that's easier to understand someone your age." Harper expresses the awareness that peers can help each other, stating "we always try to relate to them because we are the 
International Journal of Child, Youth and Family Studies (2014) 1: 24-46

same age as them." The participants agree that an important factor in the impact of the group was the peer-to-peer connections that were established.

Voice of altruism. The voice of altruism was marked by language about giving back to the world or using their experiences in the group to make an impact on others. One aspect of the altruistic voice is the inner gratification from helping that the participants described. Anne discusses how "going to schools and talking is a big thing. I love to do that. It's a good feeling inside." Similarly, Harper shares, "I can be proud of myself for doing something and teaching people stuff." Through the public activities of the Alive group, participants experienced themselves as positive agents of change.

The altruistic voice was also noted in the participants' desire to share their experience of value in the Alive group with others. In contrast with the aforementioned focus on the personal gratification felt in helping, this aspect of the voice of altruism was focused on the healing of others. For instance, Lucy shares, "I just wanted to teach other people about what I know." As Harper talks about how she would rather do presentations than be simply a support group, she shares, "[doing presentations] feels like we're making a difference and actually doing something." It appears that their own experiences with suicidality and depression gave the group members an increased amount of empathy for others who are struggling with similar concerns. Their journey through these issues seems to have fed their desire to make a difference in the lives of others.

Activist voice. We heard in one participant's interview the altruistic voice cooccurring with her literal speaking voice. When Lucy's literal voice occurred at the same time as her altruistic voice, her voice took on a unique tone. As she spoke, it was as if she was making a speech. In her interview, she even referred to herself as an activist. We decided to title this voice the activist voice. Listen as she speaks with it:

We can show them that just the four walls in the counselling office, those four walls don't have to be that small, you know what I mean? We can expand those. Like, [our high school] can be our walls to start. Everyone can talk to each other. Everyone can know. Everyone can understand each other.

This activist voice goes further than to talk about helping others; she creates the sense that she is going to revolutionize the world. As we listened to Lucy speak from this voice, we were inspired by the power in her voice and by the desire she has to shift others' way of thinking and acting towards suicide. She speaks again in this activist voice as she shares about what it is like to present:

It's those moments where you go in front of a crowd of people and everything you feel kind of different. You feel like you're the outsider, the one that's stepping kind of out of the line, and all of these kids are just watching you being like, okay, whatever. Like, sometimes it feels like that, and then as soon as you finish your presentation, it kind of feels like everybody kind of steps a little bit out of their line. 
International Journal of Child, Youth and Family Studies (2014) 1: 24-46

Here, she paints a picture of what it is like to make a difference. After experiencing the impact of openness and vulnerability in the group, she is committed to taking leadership in offering this knowledge to others. Lucy's words reflect that she does not participate in the group only because she enjoys it or likes the way it feels; the urgency in her activist voice communicates that, for her, this is something she must do.

Voice of protection. Throughout the data collection process for the present study, the participants experienced a change in leadership within their school-based group. Having experienced a profound impact in the group under the first leader with whom the members felt a strong sense of connection, the leadership change introduced a novel challenge. A voice of protection emerged within the interviews that described their reaction to this change. The participants voiced feelings of anger and frustration; listening to these, we came to hear how these were directed at the recent changes in the group, originating in a desire to protect the value of the group that they had helped to create. Demonstrating the essence of the protective voice, Jane shares, “We don't want to lose what we created." Some participants directed the protective voice at members who demonstrated less investment in the group or attended sporadically. Lucy shares: "This group is so important. It's not just a place where we meet. It's not just presenting to people. It's changing the world in our own little kind of [way]."

Some members responded to the change in leadership by not attending the Alive group. Speaking about members who had stopped attending Lucy states, "Jane and Harper don't show up anymore because they don't like [the new leader] and they don't like the new people, but we have to have new people, and it's always kind of like that battle between do we fight for it or do we grow up?" Lucy demonstrates a resilient and balanced approach to validate the frustration she and others experienced while holding on to a bigger sense of purpose and meaning.

\section{Conclusion}

The traditional format of a research article might be to now interpret the voices of the Alive group participants and to apply our perspectives as researchers, the underlying assumption being that our perspectives, as formally educated individuals, are prioritized and elevated in importance, when compared with the inexperienced or less formally educated voices of the participants. Often, the researcher is given the task of bringing the voices into context and providing a tangible summation of what these voices mean for researchers and practitioners in the world of suicide prevention education. As we reflect on this usual way of presenting research, we feel a resistance to simplifying or to attempting to encapsulate what has already been quite profoundly stated by the participants.

Perhaps a narrative of a perspective on suicide prevention will help to anchor this perspective. Chelsea recalls a story told by a colleague about an encounter within an Aboriginal community. After a number of recent completed suicides of Aboriginal youth, an elder from a particular Aboriginal community stated that an answer, from his perspective, to the problem of suicide was, to build more canoes. From an outsider 
perspective, this advice seems confusing, perhaps even unhelpful. How can building more canoes protect individuals or communities from suicidality? When probed further, the elder describes the process and meaning of making canoes within that community. When the community builds canoes, the young are with the old. They come together for a common purpose, for mutual support, with all the tradition, culture, and meaning of this common purpose surrounding the young people. Through the building of the canoes, the youth can experience purpose, meaning, and connection in a tangible, communityfocused way.

At the outset of the present study, we hoped that exploring the perspectives of the Alive members would cast new light on their experience of a suicide prevention education initiative. It is with this intent of casting new light that we hope to highlight the participants' unique perspectives through these concluding comments.

It appears that the participants were impacted primarily through the experience of connection. Through identification and connection with others who had experienced similar difficulties, the participants discovered that they are not alone. This discovery instilled hope. Through this identification, the participants were able to come to a greater understanding of their own internal experience. Their deeper understanding seemed to increase participants' sense of self-efficacy, fostering an ability to take control of their lives. The experience of connection also enabled the participants to experience vulnerability in a safe and supportive environment. Learning that relationships can provide healing seemed to enable the participants to create more healthy relationships in their own lives.

Through the connection in the Alive group, the participants came to know themselves more deeply, and they became more confident in trusting their own intuition. Becoming more familiar with who they are and who they want to be, they were better able to maintain a healthy emotional state. Though many of them still evidenced risk factors for suicidality, they became more liable to bounce back from adverse circumstances, and they knew what to do to stay well. Learning about suicidality enabled the participants to help others and to help themselves. Because they became more aware of how helping others can negatively impact their own well-being, the participants learned to ask for what they need, ensuring that they would not lose touch with their own voice for the sake of relationship. Joining each other in shared vulnerability, they affirmed each other's strengths, courageously moving closer to self-acceptance. Together, they formed a common purpose in looking outward, with a vision and means to help others around a theme with deep personal significance for each of them. Without a formal, externally imposed curriculum that would lend to proper effectiveness in research standards, the activities of the Alive group were akin to building canoes in the context of their social ecosystem. This group offered them purpose, meaning, connection, and support as valued members and as active agents of change in their community.

According to their expressed voices of experience, these adolescent girls were profoundly impacted through the period of their involvement in the group. The voices of vulnerability evidenced in this study include voices of not knowing, disconnection, and 
silence. These voices welled up in their interviews primarily as the participants discussed past experiences of suicidality and depression. References to the voices of resiliency in the participants' experiences - voices of knowing, connection, altruism, and protection were largely linked to their involvement in the Alive group. Through the connection gained in the Alive group, they experience their lives less through the voices of vulnerability, rather coming to a greater sense of knowing about themselves and others. As they experienced healing, they expressed a desire to help others and to impact the world with what they have learned.

As we have listened to the voices of these participants, what do their voices tell us about doing suicide prevention education? Resonating with the findings and perspectives of White and Morris (2010), suicide prevention initiatives that are standardized, driven by facts and content, and applied universally to all individuals miss the mark. In fact, what these participants are crying out for is to be heard and to be treated as real people, unique and valued in relationships. They do not want to be boiled down to a list of risk and protective factors, put in a category, or be the passive recipients of an intervention.

Though not formulaic, further interventions and research related to resiliency in youth can be informed by the experiences of the Alive group participants. Perhaps for the same reasons that the Alive group cannot be replicated and evaluated according to commonly accepted outcome standards for evidence-based practice, it may have worked for these participants: It was in fact alive, an organically inspired process, born out of the individually experienced pain and dynamic personalities of its participants in the context of their shared experiences. Leadership for the group created space and safety for encounters with each other, in which the participants' experienced healing and pursued activism. These participants expressed wanting to be known, have a voice in these interventions, enact the vision that they are passionate about, and be surrounded by a community that supports them and values the contribution they offer to the world. 
International Journal of Child, Youth and Family Studies (2014) 1: 24-46

\section{References}

Ciffone, J. (2007). Suicide prevention: An analysis and replication of a curriculum-based high school program. Social Work, 52, 41-49. doi: 10.1093/sw/52.1.41 http://dx.doi.org/10.1093/sw/52.1.41

Corcoran, J., Dattalo, P., Crowley, M., Brown, E., \& Grindle, L. (2011). A systematic review of psychosocial interventions for suicidal adolescents. Children and Youth Services Review, 33(11), 2112-2118. doi: 10.1016/j.childyouth.2011.06.017 http://dx.doi.org/10.1016/j.childyouth.2011.06.017

Everall, R. D., Altrows, K. J., \& Paulson, B. L. (2006) Creating a future: A study of resilience in suicidal female adolescents. Journal of Counseling and Development, 84(4), 461-470. Retrieved from http://eric.ed.gov:80/ERICWebPortal /search/detailmini.jsp?_nfpb=true\&_\&ERICExtSearch_SearchValue_0=EJ74218 6\&ERICExtSearch_SearchType_0=no\&accno=EJ742186 http://dx.doi.org/10.1002/j.1556-6678.2006.tb00430.x

Gilligan, C. (1982). In a different voice: Psychological theory and women's development. Cambridge, MA: Harvard University Press. http://dx.doi.org/10.2307/2067520

Gilligan, C., \& Machoian, L. (2002). Learning to speak the language: A relational interpretation of an adolescent girl's suicidality. Studies in Gender and Sexuality, 3(3), 321-341. http://dx.doi.org/10.1080/15240650309349204

Gilligan, C., Spencer, R., Weinberg, M. K., \& Bertsch, T. (2003). On the listening guide: A voice-centered relational model. In P. M. Camic, J. E. Rhodes, \& L. Yardley (Eds.), Qualitative research in psychology: Expanding perspectives in methodology and design (pp. 157-172). Washington, DC: American Psychological Association. http://dx.doi.org/10.1037/10595-009

Guo, B., \& Harstall, C. (2002). Efficacy of suicide prevention programs for children and youth. (Health Technology Assessment 26: Series A). Edmonton, AB: Alberta Heritage Foundation for Medical Research. Retrieved from www.ihe.ca/documents/suicide_prevention.pdf

Hooven, C., Herting, J. R., \& Snedker, K. A. (2010). Long-term outcomes for the promoting CARE suicide prevention program. American Journal of Health Behavior, 34(6), 721-736. Retrieved from http://www.atyponlink.com/PNG/doi/abs/10.5555/ajhb.2010.34.6.721 http:/dx.doi.org/10.5993/ajhb.34.6.8

Johnson, J., Wood, A. M., Gooding, P., Taylor, P. J., \& Tarrier, N. (2011). Resilience to suicidality: The buffering hypothesis. Clinical Psychology Review, 31(4), 563591. doi: 10.1016/j.cpr.2010.12.007 http://dx.doi.org/10.1016/j.cpr.2010.12.007

Leuthardt, N. (2011, June). Youth led suicide prevention education final report. Unpublished report on the Alive group. 
International Journal of Child, Youth and Family Studies (2014) 1: 24-46

Marchand, E., Stice, E., Rhode, P., \& Becker, C. B. (2011). Moving from efficacy to effectiveness trials in prevention research. Behavior Research and Therapy, 49(1), 32-41. doi: 10.1016/j.brat.2010.10.008 http://dx.doi.org/10.1016/j.brat.2010.10.008

Mikel Brown, L. (1997). Performing feminities: Listening to white working-class girls in rural Maine. Journal of Social Issues, 53(4), 683-701. http://dx.doi.org/10.1111/j.1540-4560.1997.tb02456.x

Miller, D. N., Eckert, T. L., \& Mazza, J. J. (2009). Suicide prevention programs in the schools: A review and public health perspective. School Psychology Review, 38(2), 168-188.

Nrugham, L., Holen, A., \& Sund, A. M. (2010). Associations between attempted suicide, violent life events, depressive symptoms, and resilience in adolescents and young adults. The Journal of Nervous and Mental Disease, 198(2), 131-136. doi:

10.1097/NMD.0b013e3181da48b3 http://dx.doi.org/10.1097/nmd.0b013e3181cc43a2

Rhodes, A. E., Bethell, J., \& Bondy, S. (2006). Suicidality, depression, and mental health service use in Canada. Canadian Journal of Psychiatry, 51, 35-41. Retrieved from http://ww1.cpa-apc.org:8080/publications/archives/cjp/2006/january/ rhodes-OR.asp

Richardson, G. E., Neiger, B. L., Jensen, S., \& Kumpfer, K. L. (1990). The resiliency model. Health Education, 21, 33-39. http://dx.doi.org/10.1080/00970050.1990.10614589

Rutter, M. (1987). Psychosocial resilience and protective mechanisms. American Journal of Orthopsychiatry, 57(3), 316-331. Retrieved from http://ezproxy.student.twu.ca:3446/doi/10.1111/j.1939-0025.1987.tb03541.x/pdf http://dx.doi.org/10.1111/j.1939-0025.1987.tb03541.x

Rutter, M. (2001). Psychosocial adversity: Risk, resilience and recovery. In J. M. Richman \& M. W. Fraser (Eds.), The context of youth violence: Resilience, risk, and protection (pp. 13-41). Westport, CT: Praeger.

Skinner, R., \& McFaull, S. (2012). Suicide among children and adolescents in Canada: Trends and sex differences, 1980-2008. The Canadian Medical Association Journal, 184(9), 1029-1034. doi: 10.1503/cmaj.111867 http://dx.doi.org/10.1503/cmaj.111867

Smith, A., Stewart, D., Peled, M., Poon, C., \& Saewyc, E. (2009). A picture of health: Highlights from the 2008 BC adolescent health survey. Vancouver, BC: McCreary Centre Society. Retrieved from http://www.mcs.bc.ca/ahs_reports

Tompkins, T. L., Witt, J., \& Abraibesh, N. (2010). Does a gatekeeper suicide prevention program work in a school setting? Evaluating training outcome and moderators of effectiveness. Suicide and life-threatening behavior, 40(5), 506-515. http://dx.doi.org/10.1521/suli.2010.40.5.506 
International Journal of Child, Youth and Family Studies (2014) 1: 24-46

Ungar, M. (2002). Playing at being bad: The hidden resilience of troubled teens. East Lawrencetown, NS: Pottersfield Press.

Ungar, M. (2004). Nurturing hidden resilience in troubled youth. Toronto: University of Toronto Press. http://dx.doi.org/10.1093/bjsw/bch237

Ungar, M. (2005). Introduction: Resilience across cultures and contexts. In M. Ungar (Ed.), Handbook for working with children and youth: Pathways to resilience across cultures and contexts (pp. xv-xxxix). Thousand Oaks, CA: Sage.

White, J., \& Morris, J. (2010). Precarious spaces: Risk, responsibility and uncertainty in school-based suicide prevention programs. Social Science \& Medicine, 71, 21872194. doi: 10.1016/j.socscimed.2010.09.046 http://dx.doi.org/10.1016/j.socscimed.2010.09.046

White, J., Morris, J., \& Hinbest, J. (2012). Collaborative knowledge-making in the everyday practice of youth suicide prevention education. International Journal of Qualitative Studies in Education, 25(3), 339-355.

doi: 10.1080/09518398.2010.529852 http://dx.doi.org/10.1080/09518398.2010.529852 